\title{
Concurrent Gastric and Pulmonary Mucosa-Associated Lymphoid Tissue Lymphomas with Pre-Existing Intrinsic Chronic Inflammation: A Case Report and a Review of the Literature
}

\author{
Sooyeon Oh*, Nayoung Kim ${ }^{*}{ }^{\dagger}$, Dong Hyun $\mathrm{Oh}^{\dagger}$, Soo-Mee Bang ${ }^{\dagger}$, Yoon Jin Choi ${ }^{\dagger}$, Ju Yub Lee ${ }^{\dagger}$, Kyung Won Lee ${ }^{\ddagger}$, Ho II Yoon ${ }^{\dagger}$, \\ Hee Chul Yang ${ }^{\S}$, Jin Ho Paik", Dong Ho Lee* ${ }^{*}$, , and Hyun Chae Jung* \\ ${ }^{*}$ Department of Internal Medicine and Liver Research Institute, Seoul National University College of Medicine, Seoul, Departments of ${ }^{\dagger}$ Internal \\ Medicine, ${ }^{\ddagger}$ Radiology, ${ }^{s}$ Thoracic and Cardiovascular Surgery, and "Pathology, Seoul National University Bundang Hospital, Seongnam, Korea
}

Herein, we report a rare case of concurrent gastric and pulmonary mucosa-associated lymphoid tissue (MALT) lymphomas. A 65-year-old man who had been diagnosed with Helicobacter pylori-positive gastric MALT lymphoma received eradication therapy and achieved complete remission. During follow-up, he developed de novo pulmonary MALT Iymphoma as a sequela of pulmonary tuberculosis, accompanied by recurrent gastric MALT lymphoma. Polymerase chain reaction (PCR) products of the CDR3 region of the immunoglobulin heavy chain gene showed an overall polyclonal pattern with bands at 400 base pairs (bp) and 200 bp predominant in the pulmonary tissue, as well as two distinctive bands in the gastric tissue at $400 \mathrm{bp}$ and $200 \mathrm{bp}$. This case suggests that multiorgan lymphomas are more likely to be independent from each other when they are far apart, involve different organ systems, and have independent precipitating factors. (Gut Liver 2015;9:424-429)

Key Words: Stomach; Lung; Lymphoma, B-cell, marginal zone

\section{INTRODUCTION}

Mucosa-associated lymphoid tissue (MALT) lymphoma can arise from extranodal sites in virtually any organ but usually remains in the primary organ. Therefore, MALT lymphoma with multiorgan involvement had been considered to be either disseminated or relapsed disease. ${ }^{1}$ Until a few decades ago, it was difficult to prove that synchronous or metachronous MALT lymphomas of multiple organs were independent. However, polymerase chain reaction (PCR) and other sequencing methods have made it easier to elucidate whether lesions are clonal. So far, there have been 16 such attempts. Here, we present a case of multiorgan MALT lymphoma, consisting of a gastric MALT lymphoma related to a current Helicobacter pylori infection and a pulmonary MALT lymphoma that was related to previous infection with Mycobacterium tuberculosis. We attempted to investigate the clonality of the two MALT lymphomas through PCR analysis of the CDR3 region of the immunoglobulin heavy chain gene $(\mathrm{IgH})$.

\section{CASE REPORT}

A 65-year-old man who had undergone unsuccessful treatment for a gastric ulcer was referred to our hospital. He had been treated for pulmonary tuberculosis 40 years ago. He was an ex-smoker with a 15 pack-year smoking history. The only discomfort he had was postprandial nausea. Physical examination revealed mild inspiratory crackles in the right upper lung field that were caused by previous pulmonary tuberculosis. Otherwise, there were no remarkable findings upon physical examination. Gastric endoscopy showed a slightly depressed lesion with irregular nodularity at the posterior wall of the gastric mid to high body (Fig. 1A). MALT lymphoma was suspected, and multiple biopsies were performed. Hematoxylin and eosin staining of the biopsied tissues showed that the mucosa was invaded by small lymphocytes forming lymphoepithelial lesions, and $H$. pylori was present (Fig. 1B). On immunohistochemical staining, $\mathrm{CD} 20$ was positive, $\mathrm{CD} 3$ was focally positive, $\mathrm{Ki}-27$ was positive in $1 \%$ to $2 \%$, and cytokeratin staining revealed the presence of lymphoepithelial lesions (Fig. 1C-F). Thus, the pathologic findings were consistent with a low-grade extranodal marginal zone B-cell MALT lymphoma. API2/MALT1 translocation $\mathrm{t}(11 ; 18)$

Correspondence to: Nayoung Kim

Department of Internal Medicine, Seoul National University Bundang Hospital, 82 Gumi-ro 173beon-gil, Bundang-gu, Seongnam 463-707, Korea

Tel: +82-31-787-7008, Fax: +82-31-787-4051, E-mail: nayoungkim49@empas.com

Received on February 23, 2014. Revised on April 21, 2014. Accepted on June 26, 2014.

pISSN 1976-2283 eISSN 2005-1212 http://dx.doi.org/10.5009/gnl14072

(c) This is an Open Access article distributed under the terms of the Creative Commons Attribution Non-Commercial License (http://creativecommons.org/licenses/by-nc/3.0) which permits unrestricted non-commercial use, distribution, and reproduction in any medium, provided the original work is properly cited. 

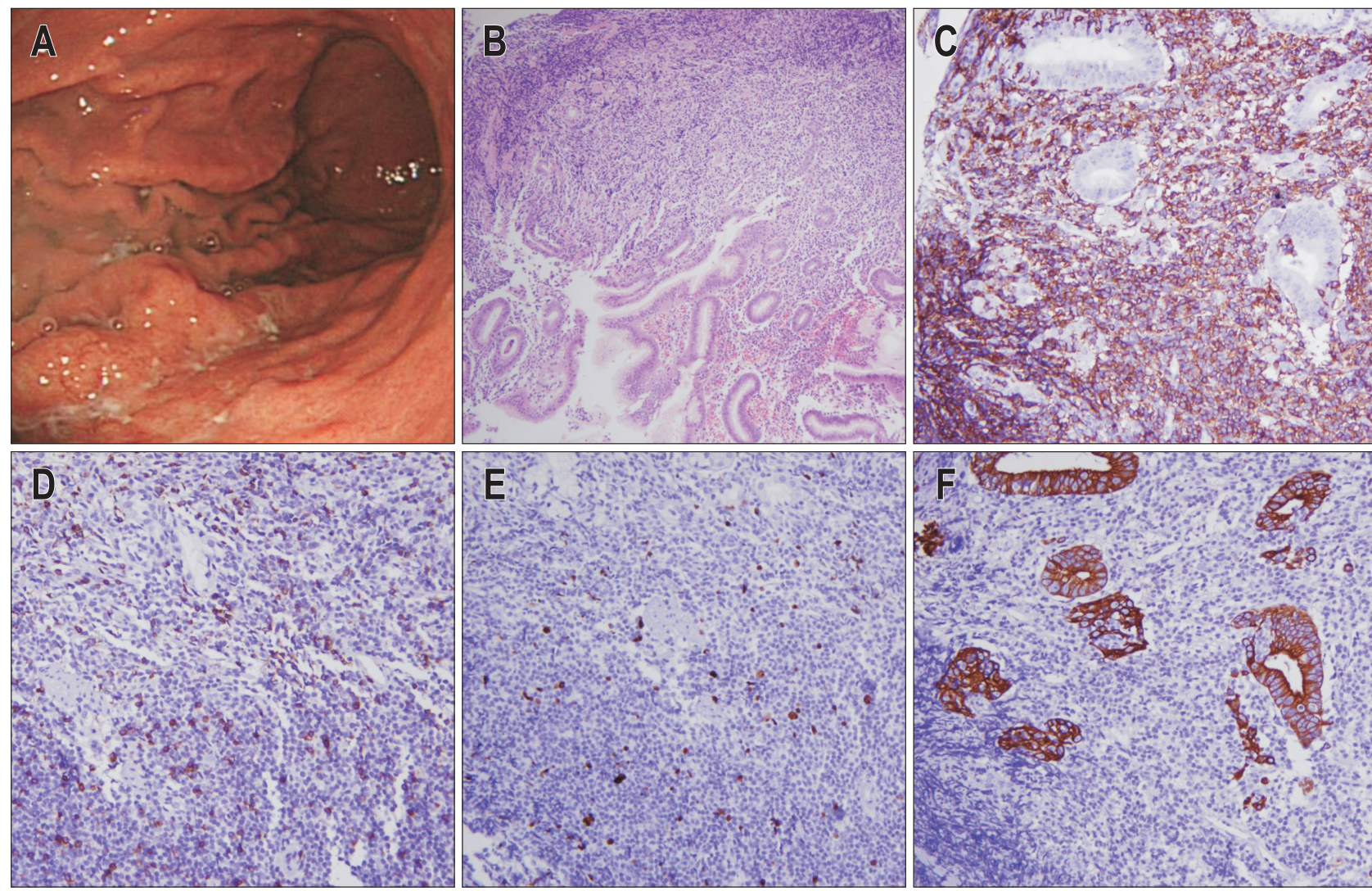

Fig. 1. A slightly depressed lesion with irregular nodularity at the posterior wall of the gastric mid to high body (A). Endoscopically biopsied tissues of the lesion stained with hematoxylin and eosin (×200) (B). Immunohistochemical staining showed that CD20 was positive (C), CD3 was only focally positive (D), Ki-27 was positive in $1 \%$ to $2 \%$ (E), and cytokeratin was positive (F).

(q21;q21) was detected by fluorescence in situ hybridization.

To stage the lymphoma, computed tomography (CT) of the chest was performed. In both of the upper lobes of the lung, consolidative lesions with patchy ground-glass opacities and calcifications were noticed (Fig. 2B). When the lesions were compared to a previous chest CT taken 1 year prior (Fig. 2A), there was no interval change in shape or size. Therefore, we concluded that the pulmonary lesions were sequelae of past pulmonary tuberculosis. Otherwise, there were no significant findings that would suggest nodal involvement or distant metastasis. Thus, the lymphoma was staged as IE disease. Triple therapy with a proton pump inhibitor, amoxicillin, and clarithromycin was administered for $H$. pylori eradication; 6 weeks later, a urea breath test was negative for $H$. pylori. Follow-up gastric endoscopy was performed at a 3-month interval. At the 1-year follow-up, the gastric lesion had resolved, and biopsies were negative for malignancy, signifying complete remission.

The patient returned for follow-up 6 months later (21 months after the first diagnosis of MALT lymphoma). He was in good general condition and had no discomfort or abnormal findings on physical examination, except for mild inspiratory crackles in the right upper lung field that had been previously noted. Reevaluation of disease status was performed with gastric endos- copy and a CT scan of the chest. Gastric endoscopy showed a nodular lesion at the location of the original MALT lymphoma, and two new proximal erosions were present. Biopsy specimens showed infiltration of malignant lymphoid cells within the lymphoepithelial lesion. The malignant cells were both CD20- and cytokeratin-positive, demonstrating recurrent MALT lymphoma, but $H$. pylori was not detected. CT scan showed that the previously observed consolidative lesions of the bilateral upper lung lobes had increased in extent and density (Fig. 2C). To diagnose the lung lesions, bronchoscopic washings were performed. Acidfast smears, cultures, PCR for M. tuberculosis, and cytology studies were performed on the specimen, but the results of these tests were nondiagnostic. Therefore, CT-guided percutaneous needle aspiration of the right upper lobe lesion was performed twice, but the sampled tissues showed only inflammatory cells and were not sufficient for diagnosis. Video-assisted thoracoscopic wedge resection was then performed to remove the nodular lesions in the left upper lobe. Pathologic examination of the acquired tissue showed that the alveoli were infiltrated with small lymphocytes. Immunohistochemical staining was positive for $\mathrm{CD} 20$, focally positive for $\mathrm{CD} 3,1 \%$ positive for $\mathrm{Ki}$ 67, positive for cytokeratin and BCL-2, and negative for $\mathrm{CD} 5$, CD10, CD23, BCL-6, and cyclin D1. The lesion was consistent 

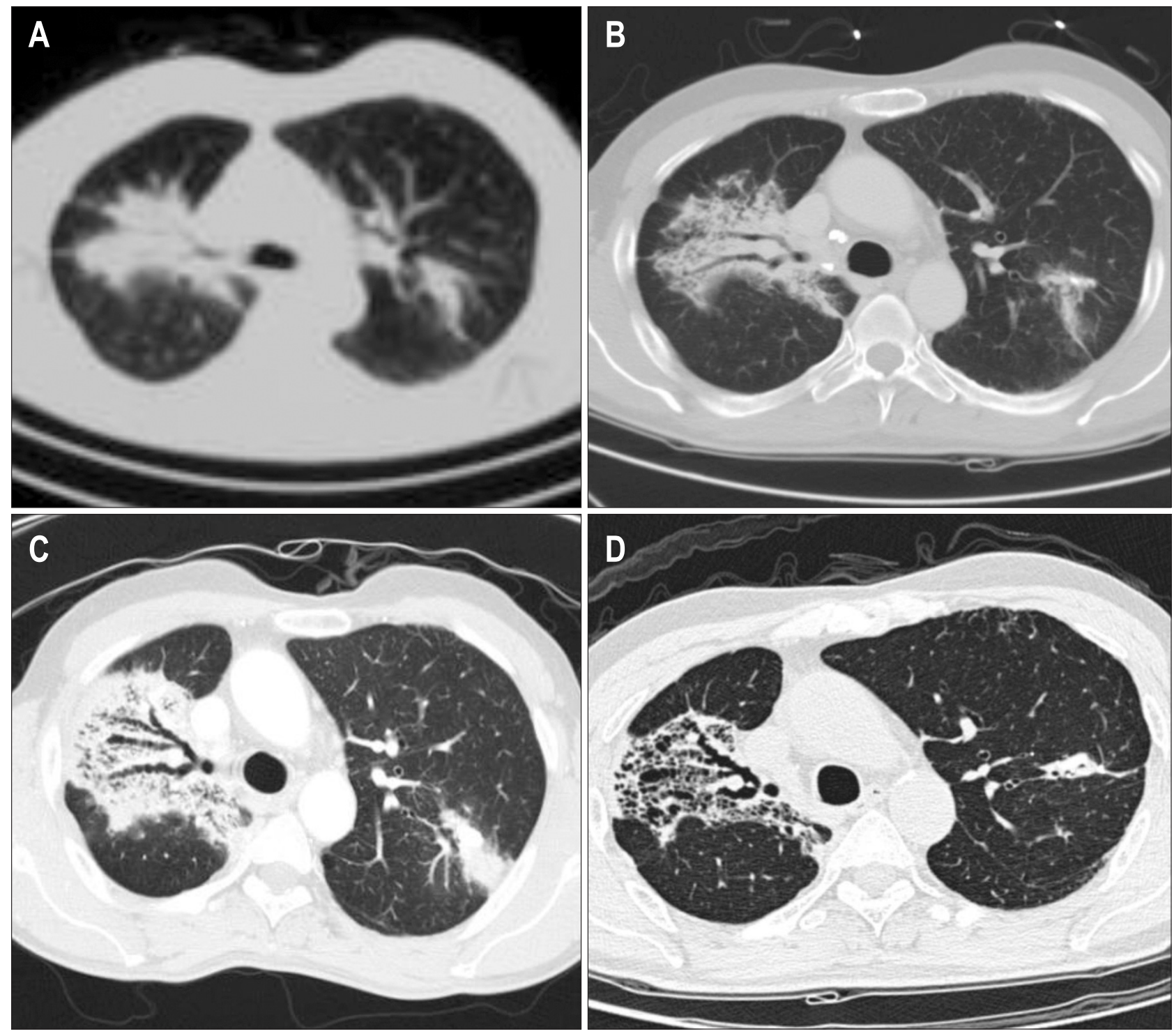

Fig. 2. The initial evaluation showed consolidative lesions with patchy ground-glass opacities and calcifications (B). When the lesions were compared to a previous chest computed tomography from 1 year prior (A), there was no interval change in the shape or size. At the 9-month followup, the consolidative lesions of both of the upper lobes had increased in their extent and density (C). After eight cycles of immunochemotherapy, the lesions had resolved (D).

with MALT lymphoma, and $\mathrm{t}(11 ; 18)(\mathrm{q} 21 ; \mathrm{q} 21)$ was present. A subsequent positron emission tomography scan showed hypermetabolic areas in the bilateral upper lobes of the lung, but no other abnormal signals suggestive of malignancy were observed (Fig. 3). Bone marrow examination showed evidence of lymphomatous involvement. The serum lactate dehydrogenase level was $277 \mathrm{IU} / \mathrm{mL}$ (reference range, 100 to $255 \mathrm{IU} / \mathrm{mL}$ ).

The lymphomas were believed to be stage IE gastric MALT lymphoma combined with stage IIE pulmonary MALT lymphoma or stage IV gastric MALT lymphoma with pulmonary dissemination. The absence of lymph node or bone marrow involvement seemed to indicate the former. For further investigation, PCR of the CDR3 region of IgH was performed with formalin-fixed paraffin-embedded tissue samples, according to previously published methods. ${ }^{2,3}$ The following consensus primers, which were specific for CDR3, were used: the first-round primer was LJH 5'-TGAGGAGACGGTGACC-3'; and the nested second-round primers were VLJH 5'-GTGACCAGGGTNCCTTGGCCCCAG-3', and FR3A 5'-ACACGGCSSTGTATTACTGT-3'. Electrophoresis of the PCR products showed an overall polyclonal pattern in the pulmonary tissues, even though bands at 400 base pairs (bp) and $200 \mathrm{bp}$ predominated. Two distinctive bands at $400 \mathrm{bp}$ and $200 \mathrm{bp}$ were observed in the gastric tissues (Fig. 4).

Chemotherapy was administered, considering the recurrent gastric MALT lymphoma combined with pulmonary MALT lymphoma. The patient received eight cycles of immunochemotherapy with rituximab, cyclophosphamide, vincristine, and 


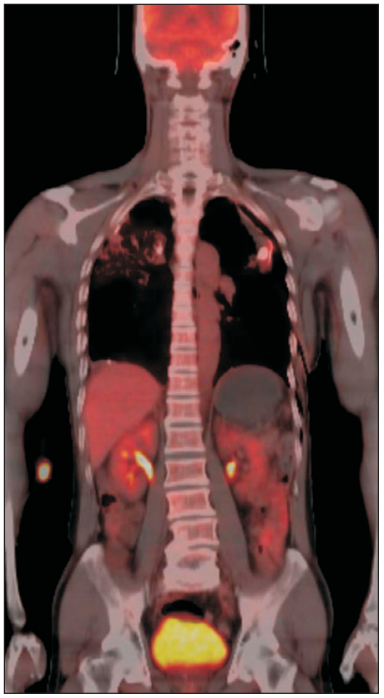

Fig. 3. The positron emission tomography scan revealed hypermetabolic signals in both upper lobes of the lung, but no other abnormal signals suggestive of malignancy were observed.

prednisolone. Upon completion of the immunochemotherapy, follow-up evaluations were performed. Gastric endoscopy showed healed scars at the original location of the gastric MALT lymphoma, and biopsies from the scars were compatible with chronic gastritis with intestinal metaplasia. CT scan also showed resolution of the lung lesions (Fig. 2D). Thus, the patient achieved complete remission again. We plan to follow him regularly.

\section{DISCUSSION}

Our patient is a good example of the influence of infection and chronic inflammation on the development of MALT lymphoma. The patient was positive for $H$. pylori and thus was thought to have developed gastric MALT lymphoma due to $H$. pylori infection. Initially, $H$. pylori eradication followed by a "watch and wait" strategy was chosen because it is the mainstay of stage I gastric MALT lymphoma treatment. Unfortunately, gastric MALT lymphoma recurred, which may have been predicted by the lesion's positive $t(11 ; 18)(q 21 ; q 21)$ status, which increases the risk of nonresponsiveness to $H$. pylori eradication therapy and relapse compared to $t(11 ; 18)(q 21 ; q 21)$ negative patients. ${ }^{4}$ In this case, the recurrence of gastric MALT lymphoma was accompanied by pulmonary MALT lymphoma. Because the pulmonary MALT lymphoma developed in a background of tubercular sequelae, and there was no nodal involvement to suggest disseminated disease, we believe that the pulmonary MALT lymphoma is likely to have developed de novo and that chronic inflammation due to past infection with $M$. tuberculosis may have contributed to the development of lymphoma.

Chronic inflammation caused by either persistent infection or an autoimmune disorder precipitates the formation of orga-

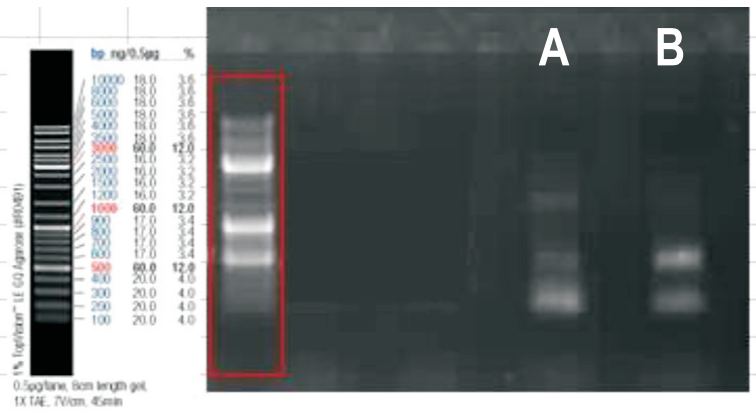

Fig. 4. Polymerase chain reaction (PCR) of the CDR3 region of immunoglobulin heavy chain gene was performed with formalin-fixed paraffin-embedded tissue samples. Electrophoresis of the PCR products demonstrated an overall polyclonal pattern with predominant bands at 400 base pairs (bp) and $200 \mathrm{bp}$ in the pulmonary tissues (A), while two distinctive bands at $400 \mathrm{bp}$ and $200 \mathrm{bp}$ were noted in the gastric tissues (B).

nized lymphoid tissue and a microenvironment that facilitates lymphomagenesis. Chronic inflammation results in persistent antigenic or auto-antigenic stimulation, which in turn triggers polyclonal B-cell proliferation and recruits a series of inflammatory cells, including T-lymphocytes, macrophages and neutrophils. The resulting microenvironment promotes the growth of neoplastic B-lymphocytes, allowing the acquisition of genetic aberrations and proliferation. ${ }^{5}$ Some of these relationships have already been thoroughly elaborated in review articles, such as the relationship of $H$. pylori to gastric MALT lymphoma, that of Chlamydia psittaci to ocular adnexa MALT lymphoma, that of Borrelia burgdorferi to cutaneous MALT lymphomas, that of Sjögren's syndrome to salivary gland MALT lymphoma, that of Hashimoto's thyroiditis to thyroid MALT lymphoma, and so forth. ${ }^{5}$

The best known of these relationships is the association between infection with $H$. pylori and gastric MALT lymphoma. In previous studies, $88.8 \%$ of gastric MALT lymphoma patients had H. pylori ${ }^{6}$ infections; approximately $75 \%$ of these patients achieved complete remission after $H$. pylori eradication alone. ${ }^{4,7-10}$ The pathogenic relationship between MALT lymphoma and $H$. pylori infection remains unclear. However, it has been postulated that elevated levels of some cytokines in the tumor necrosis factor family are produced by macrophages that are present in MALT; these cytokines may play a crucial role in B-cell transformation and progression into malignant lymphoma, in concert with additional signals from $\mathrm{T}$ cells or direct antigenic autostimulation. ${ }^{11}$

Although rare, there have been a few reports suggesting an association between infections with Mycobacterium species and pulmonary MALT lymphoma. ${ }^{12-15}$ In two of these reports, pulmonary MALT lymphoma developed during follow-up after the successful treatment of active mycobacterial pulmonary infections. In another case, pulmonary tuberculosis and pulmonary MALT lymphoma were synchronously diagnosed. In the other 
case, pulmonary MALT lymphoma developed in a patient with a remote history of tuberculosis, as in the present case. In mycobacterial infections, inflammation is mediated by interferon- $\gamma$, which is produced by antigen-activated $\mathrm{T}$ cells and in turn stimulates macrophages. $\mathrm{T}$ cells and macrophages produce other inflammatory cytokines and can promote B-cell proliferation, thereby aiding lymphomagenesis. ${ }^{12}$ The present case suggests that it may be reasonable to consider the possibility that chronic inflammation triggered by $M$. tuberculosis may persist long after successful treatment.

In the setting of chronic inflammation, multiple B-cells proliferate; dominant clones appear and transform into lymphoma as the disease advances. ${ }^{16}$ Our PCR results demonstrated an overall polyclonal pattern with predominant bands at $400 \mathrm{bp}$ and $200 \mathrm{bp}$ in the pulmonary tissue, and two distinctive bands at $400 \mathrm{bp}$ and $200 \mathrm{bp}$ were observed in the gastric tissue (Fig. 4). These results suggest that we captured two different phases of lymphomagenesis. Gastric MALT lymphoma occurred almost 2 years earlier than pulmonary MALT lymphoma; this timing is consistent with the fact that gastric MALT lymphoma had already developed dominant clones, while the pulmonary MALT lymphoma was still in the polyclonal phase. We cannot exclude the possibility that these two lymphomas may be clonally related, as they both showed predominance in $400 \mathrm{bp}$ and $200 \mathrm{bp}$. However, the fact that the pulmonary MALT lymphoma had a polyclonal background suggests that it developed independently. If the pulmonary MALT lymphoma had disseminated from the gastric MALT lymphoma, it would not have had a polyclonal background. Sequencing is required to demonstrate that the two MALT lymphomas were independent. We proceeded to perform sequencing. However, doing it without the cloning process which is quite expensive, we failed to acquire exact sequences. PCR and sequencing are excellent tools for the detection of clonality, and this information could be crucial in the staging of lymphomas with multiorgan involvement in the absence of nodal or bone marrow involvement. However, PCR and sequencing cannot be recommended to patients routinely because the clinical significance of these tests has not yet been determined. In the present case, detecting clonality did not change the treatment plan and only helped to predict the prognosis. Performing expensive tests only to obtain prognostic information does not seem practical. Therefore, we would not recommend PCR testing at the patient's expense; however, for academic purposes, we performed the test free of charge with the patient's consent.

Nonetheless, we can utilize information from previous studies to reach a working conclusion regarding clonality and prognosis. When the two involved organs are in proximity, for example, the ocular adnexa and the parotid gland, or within the same organ system, for example, the stomach and intestine, there is a higher probability that the two lymphomas are from the same clone, indicating dissemination and therefore a poor prognosis. ${ }^{15,17}$ When the two involved organs are distant from each other or in different organ systems, as in the present case, the two lymphomas are more likely to have developed independently. Another clinical cue indicating that our patient had two independent MALT lymphomas is that each organ had independent precipitating factor, that is, the stomach had chronic inflammation due to $H$. pylori and the lung had that due to prior infection of $M$. tuberculosis.

Although the conclusion above remains to be confirmed since it is based on only a handful of cases, the explanation is reasonable. More data need to be accumulated to present a guideline for diagnosing and treating multiorgan MALT lymphomas. For now, when MALT lymphomas with multiorgan involvement are encountered, it is best to analyze the case in its context, use all of the available information, and determine the most suitable evaluation and treatment plan.

\section{CONFLICTS OF INTEREST}

No potential conflict of interest relevant to this article was reported.

\section{ACKNOWLEDGEMENTS}

This work was supported by the National Research Foundation of Korea (NRF) grant for the Global Core Research Center (GCRC) funded by the Korea government (MSIP) (number: 20110030001).

\section{REFERENCES}

1. Thieblemont C, Berger F, Dumontet C, et al. Mucosa-associated lymphoid tissue lymphoma is a disseminated disease in one third of 158 patients analyzed. Blood 2000;95:802-806.

2. Diss TC, Peng H, Wotherspoon AC, Isaacson PG, Pan L. Detection of monoclonality in low-grade B-cell lymphomas using the polymerase chain reaction is dependent on primer selection and lymphoma type. J Pathol 1993;169:291-295.

3. Iwano M, Okazaki K, Uchida K, et al. Characteristics of gastric Bcell lymphoma of mucosa-associated lymphoid tissue type involving multiple organs. J Gastroenterol 2004;39:739-746.

4. Choi YJ, Kim N, Paik JH, et al. Characteristics of Helicobacter pylori-positive and Helicobacter pylori-negative gastric mucosaassociated lymphoid tissue lymphoma and their influence on clinical outcome. Helicobacter 2013;18:197-205.

5. Thieblemont C, Bertoni F, Copie-Bergman C, Ferreri AJ, Ponzoni M. Chronic inflammation and extra-nodal marginal-zone lymphomas of MALT-type. Semin Cancer Biol 2014;24:33-42.

6. Zullo A, Hassan C, Cristofari F, et al. Effects of Helicobacter pylori eradication on early stage gastric mucosa-associated lymphoid tissue lymphoma. Clin Gastroenterol Hepatol 2010;8:105-110.

7. Bayerdörffer E, Neubauer A, Rudolph B, et al. Regression of pri- 
mary gastric lymphoma of mucosa-associated lymphoid tissue type after cure of Helicobacter pylori infection. MALT Lymphoma Study Group. Lancet 1995;345:1591-1594.

8. Nakamura S, Matsumoto T, Suekane H, et al. Long-term clinical outcome of Helicobacter pylori eradication for gastric mucosaassociated lymphoid tissue lymphoma with a reference to secondline treatment. Cancer 2005;104:532-540.

9. Ruskoné-Fourmestraux A, Fischbach W, Aleman BM, et al. EGILS consensus report: gastric extranodal marginal zone B-cell lymphoma of MALT. Gut 2011;60:747-758.

10. Wotherspoon AC, Doglioni C, Diss TC, et al. Regression of primary low-grade B-cell gastric lymphoma of mucosa-associated lymphoid tissue type after eradication of Helicobacter pylori. Lancet 1993;342:575-577.

11. Witkowska M, Smolewski P. Helicobacter pylori infection, chronic inflammation, and genomic transformations in gastric MALT lymphoma. Mediators Inflamm 2013;2013:523170.

12. Inadome $Y$, Ikezawa $T$, Oyasu R, Noguchi M. Malignant lymphoma of bronchus-associated lymphoid tissue (BALT) coexistent with pulmonary tuberculosis. Pathol Int 2001;51:807-811.
13. Gaur S, Trayner E, Aish L, Weinstein R. Bronchus-associated lymphoid tissue lymphoma arising in a patient with bronchiectasis and chronic Mycobacterium avium infection. Am J Hematol 2004;77:22-25.

14. Klein TO, Soll BA, Issel BF, Fraser C. Bronchus-associated lymphoid tissue lymphoma and Mycobacterium tuberculosis infection: an unusual case and a review of the literature. Respir Care 2007;52:755-758.

15. Wang Y, Dong S, Jiang Z, Li A. A case report of concurrent gastric and pulmonary mucosa-associated lymphoid tissue lymphomas and review of the literature on clonality analysis. Ann Hemato 2013;92:1707-1709.

16. Yamauchi A, Tomita Y, Miwa H, Sakamoto H, Sugiyama H, Aozasa K. Clonal evolution of gastric lymphoma of mucosa-associated lymphoid tissue type. Mod Pathol 2001;14:957-962.

17. Konoplev S, Lin P, Qiu X, Medeiros LJ, Yin CC. Clonal relationship of extranodal marginal zone lymphomas of mucosa-associated lymphoid tissue involving different sites. Am J Clin Pathol 2010; 134:112-118. 